\title{
Uber die Gliederung der Würm-Eiszeit und die Stellung der Lösse in ihr ${ }^{1}$ )
}

\author{
Von Paul Woldstedt, Bonn
}

\author{
Mit 1 Abbildung im Text
}

$\mathrm{Zus}$ a m me $\mathrm{n}$ a ssu $\mathrm{ng}$. Die Würm-Eiszeit (Weichsel-Eiszeit in Norddeutschland) gliedert sich in drei Abschnitte: Früh-, Mittel- und Spätwürm. Das Frü hw ü r m umfaßt den ersten, in sich wahrscheinlich wieder gegliederten Vorstoß des Eises bis zum Stettiner Stadium in Norddeutschland bzw. einem später überfahrenen Stadium im alpinen Gebiet. Diesem Frühwürm gehört der untere Jüngere Löß an, der gelegentlich wieder untergegliedert ist („Jüng. Löß I“). Urgeschichtlich ist das Frühwürm durch die Moustier-Industrien verschiedener Prägung charakterisiert. Das $\mathrm{M}$ i t te $\mathrm{lw}$ ü $\mathrm{rm}$ beginnt mit einem längeren, wärmeren Interstadial („Göttweiger" oder "Fellabrunner Interstadial"), in dem das Eis sehr weit zurückgegangen sein muß. Dann stieß es zur Maximalausdehnung vor, wobei eine stärkere, gelegentlich wieder unterbrochene Lößbildung erfolgte ("Jüng. Löß II"). Urgeschichtlich wird das Mittelwürm durch das Aurignac (im weiteren Sinne, d.h. einschließlich von Châtelperron, Gravette u.s.w.), ferner durch die Szeleta- und Solutré-Industrien charakterisiert. Der letzte Abschnitt, das $\mathrm{S} p$ ä t w ü $\mathrm{rm}$, umfaßt den bisher meist als "Spätglazial“ bezeichneten Abschnitt, der in Norddeutschland von dem Zurückgehen des Eises vom Pommerschen Stadium an gerechnet wird. In ihm erfolgt nur ganz lokal noch Lößbildung. Urgeschichtlich gehören in diesen Abschnitt das Magdalen und die mit diesen gleichaltrigen Kulturen (z. B. die Hamburger Stufe und dgl.).

A b s t r a ct. The Würm Glaciation (Weichsel Glaciation of Northern Germany) can be divided into three parts: Early, Middle, and Late Würm. The E a rly W ü rm comprehends the oncoming glaciation on to the - afterwards overridden - Stettin stadial in Northern Germany, and to some overridden stadials of the Subalpine Plain. The (locally twofold?) lower Younger Loess belongs to this Early Würm, which contains the varions Mousterian industries. The Midd le W ü r m begins with a pronounced warmer interstadial („Göttweig" or "Fellabrunn Interstadial"). The ice margin seems to have widely recessed. Afterwards it advanced to the Würm maximum. The upper Younger Loess, locally subdivided, was deposited. The Aurignacian (sensu lato), the Szeletian and Solutrian are characteristic for this Middle Würm. The L a t e W ü rm comprehends the ice recession from the Pommeranian to the Salpausselkä stadial (i. e. the so called "Late Glacial“). The Magdalenian and the cultures of the same age ("Hamburger Stufe" etc.) belong to this part of the Würm Glaciation.

Zwei Ansichten stehen sich heute in bezug auf den würmeiszeitlichen Löß scharf gegenüber: nach der einen, die von J. Büdel (1950), H. Freising (1951), F. WeIdenBach (1955) und anderen vertreten wird, ist nur e i n, nicht weiter gegliederter Würmlöß vorhanden, während die andere Ansicht, in der Hauptsache begründet durch W. SoERgel (1919), mit einem mehrfach gegliederten Löß der Würm-Eiszeit rechnet. SoERGEL verknüpfte die ältere Abteilung des Würmlösses, seinen Jüngeren Löß I, in Süddeutschland mit der sog. "Größten Vergletscherung“, in Norddeutschland mit dem Warthe-Stadium. Es besteht heute kein Zweifel darüber, daß sowohl die „Größte Vergletscherung“ im alpinen Bereich wie das Warthe-Stadium in Norddeutschland nicht in die Würm-Eiszeit gehören, sondern in die vorhergehende Riß- oder Saale-Eiszeit. Die scheinbar selbstverständliche Folgerung, die von BüdeL, WeIDENBACH und anderen daraus gezogen wurde, war die, daß dann auch der sog. Jüngere Löß I in die Riß-Eiszeit gehöre. Das träfe zu, wenn der einwandfreie Beweis für die Verknüpfung des Jüngeren Lösses I mit der „Größten Vergletscherung“ bzw. dem Warthe-Stadium erbracht werden könnte. In Norddeutschland kann von solch einem Nachweis keine Rede sein, und auch in Süddeutschland scheint mir dieser Beweis bisher nicht gelungen zu sein.

Lösse und Vereisungen sind ja überhaupt sehr schwer miteinander zu parallelisieren. Sie schließen einander in ihrer Verbreitung aus; meist liegt sogar ein größerer Zwischen-

1) Zusammenfassung und Erweiterung von Diskussionsbemerkungen, die auf der Tagung der Quartärvereinigung in Laufen (4. 9.) und Wien (9. 9. 1956) gemacht wurden. 
raum zwischen ihren Verbreitungsgebieten. Es gelingt aber auf einem anderen Wege, zu einer Einsicht in die Dinge zu kommen, d. h. wir müssen dem Wege folgen, den vor allem wieder SoERgel gewiesen hat: wir müssen die Faunen und Floren, aber heute auch die paläolithischen Kulturen, soweit sie sich sicher bestimmen lassen, auswerten, selbstverständlich unter Berücksichtigung aller morphologischen und stratigraphischen Gegebenheiten.

Am längsten bekannt sind die Dinge in Frankreich. In Hunderten von Profilen, und zwar sowohl in Lössen wie in Höhlen, ist festgestellt worden, daß in einer einheitlichen Schichtenfolge, die ohne Ausnahme stets nur eine kalte Fauna führt, zuunterst das Moustier (verschiedener Ausbildung), dann das Aurignac (im weitesten Sinne, d. h. das Périgord bzw. Châtelperron, das eigentliche Aurignac und das Gravette), dann das Solutré und schließlich das Magdalen auftritt. Die Zusammensetzung der Fauna wechselt wohl im einzelnen etwas, aber ste t $\mathrm{s}$ is $\mathrm{t} \mathrm{der} \mathrm{Ges}$ a $\mathrm{m} \mathrm{t} \mathrm{ch}$ a ra k ter kalt oder zum mindesten kühl ${ }^{2}$ ). Vor allem gibt es unter Hunderten von Profilen auch nicht ein einziges, wo etwa zwischen dem Moustier und dem Aurignac eine echte warme Fauna mit Elephas antiquus oder ähnlichen Tieren oder eine entsprechende interglaziale Flora aufträte. Das aber müßte gefordert werden, wenn eine echte Interglazialzeit zwischen Moustier und Aurignac vorhanden wäre.

Daß das „Kalte Moustier“ in den ersten Teil der Letzten Eiszeit gehört und nicht in die vorletzte Kaltzeit, geht auch aus den Profilen an der französischen atlantischen Küste hervor. Uber den Strandbildungen der Letzten Interglazialzeit, die an zahlreichen Punkten der Kanalküste und der übrigen atlantischen Küste vorhanden sind (vgl. u. a. G. Dubors 1924, A. Bigot 1930), treten vielfach Solifluktionsbildungen auf, die also in die beginnende Würm-Eiszeit gehören. Aus ihnen werden des öfteren Moustier-Werkzeuge angegeben.

Der französische Löß wird seit langem in einen Alteren und einen Jüngeren Löß gegliedert (vgl. die älteren Arbeiten von M. Ladriere 1890, Agafonoff \& MalychefF 1929, Breuil \& Koslowski 1931, 1932 sowie die neue Arbeit von F. Bordes 1954). Der Löß ist in einer breiten Zone vorhanden, die Belgien und Nordfrankreich umfaßt (vgl. Dubois \& Firtion 1936). Da die Bezeichnungen für das Verständnis der französischen Arbeiten wichtig sind, seien sie hier kurz angeführt. Die oberste Schicht wird (abgesehen vom "limon de lavage" $=$ Schwemmlöß) gewöhnlich als "terre à briques" $=$ Lößlehm bezeichnet. Sie ist hervorgegangen aus dem „ergeron" oder „Loess récent", unserem Jüngeren Löß. Dieser ist meist durch Geröllagen ("cailloutis“) und Verlehmungen untergegliedert. Die letztinterglaziale, vielfach rotbraune Verwitterungsschicht des Alteren Lösses wird meist als „limon rouge fendillé" oder auch einfach als „argile rouge“, der eigentliche Altere Löß (Riß) als „limon doux à points noirs“ bezeichnet. Auch dieser erweist sich öfter als untergegliedert. Gelegentlich treten noch ältere Lösse (Mindel usw.) auf.

In dem Profil, das Breuil \& Koslowski von der Grube Bultel-Tellier bei St. Acheul geben, beginnt (von unten) der Altere Löß mit einem „limon doux à points noirs“, d.h. einem ersten Rißlöß, der ein „Acheul V“ enthält. Darüber folgt ein zweiter, frischer Rißlöß, z.T. mit großen Lößkindeln, der von einem Verwitterungslehm bedeckt wird. Es handelt sich um einen rötlichen, sandigen Lehm mit zahlreichen Klüften („limon rouge fendillé"), der als Verwitterungsprodukt in der Letzten Interglazialzeit entstand. In seinem oberen Teil fand sich „Acheul VI“ und „VII“ ("Micoque“), das ja auch sonst in der Riß-WürmInterglazialzeit auftritt.

Der Jüngere Löß beginnt in der Grube Bultel-Tellier mit einem caillouti (Solifluktionshorizont), der ein "Levallois V“ (nach der Bezeichnung von BREUIL \& KosLowsKI; zum Moustier-Komplex gehörig), zusammen mit Elephas primigenius, Rhinoceros tichorhinus und Rangifer enthält. Es folgt ein Jüngerer Löß I ("ergeron“), der durch einen

$\left.{ }^{2}\right)$ Entscheidend sind nicht die eurythermen, indifferenten Formen, sondern die stenothermen. 
caillouti zweigeteilt ist und oben eine schwache rötliche Verlehmungszone zeigt. Auch die-

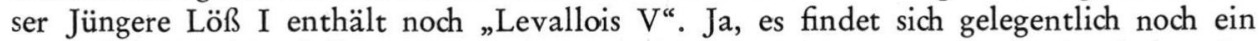
„Levallois VI“ (ebenfalls zum Moustier-Komplex gehörend) in dem caillouti, mit dem der Jüngerer Löß II beginnt. Dieser ist wieder als kalkhaltiger gelber Löß entwickelt und ist seinerseits wieder von einer Verlehmungsrinde ("terre à briques“) bedeckt. Der Jüngere Löß II bzw. seine Verlehmungsrinde enthält überall in Frankreich die Aurignac- und gelegentlich die Solutré-Kultur. Das Magdalen liegt für gewöhnlich oberhalb des Lösses.

Der Jüngere Löß des Seine-Gebietes läßt nach F. Bordes (1954) vielfach eine Dreiteilung erkennen. Ein Jüngerer Löß I enthält an seiner Basis öfter eine begrabene Schwarzerde. $\mathrm{Ob}$ es sich hierbei um einen fossilen Boden in situ, einen Prärieboden, handelt, wie BORDES meint, oder ob z.T. auch ein umgelagerter Boden vorliegt, wie H. FrEISING (1951) für viele süddeutsche Lößböden nachwies, muß offen bleiben. An Industrien tritt an der Basis des Jüngeren Löß I meist ein „Moustérien de tradition acheuléenne“ auf. Uber der schwachen Verlehmungszone des Jüngeren Löß I folgt ein Jüngerer Löß II, der ebenfalls an seiner Basis gelegentlich Moustier-Industrien enthält. Der Jüngere Löß III läßt öfter eine Zweiteilung erkennen, wobei als trennende Schicht eine Geröllage auftritt. Nach BORDES soll an der Basis des Jüngeren Löß IIIa das allerjüngste Moustier und in engstem Zusammenhang damit das älteste Périgord vorkommen. An der Basis des Jüngeren Lösses IIIb tritt dann Périgord III und in der Masse des darüber liegenden Lösses ein Périgord IV-V auf. In der höchsten, verlehmten Partie fand sich gelegentlich ein Proto-Solutré. Der Vergleich dieser Einteilung mit der von BreuIL \& KosLowsKI (1931) zeigt, daß die Lösse I + II von Bordes dem Löß I von BReul \& KosLowsKI, Löß III von Bordes dem Löß II von $\mathrm{Br} . \& \mathrm{~K}$. entsprechen.

Es erweist sich also, daß in Frankreich der Jüngere Löß mehrfach gegliedert ist, d.h. daß wir Unterbrechungen der Lößbildung gehabt haben, und daß als menschliche Kulturen das Moustier und der größte Teil des Jungpaläolithikums auftreten - stets begleitet von einer kalten Fauna, ohne daß irgendwann einmal eine echt interglaziale Fauna oder Flora dazwischen auf träte.

Dasselbe haben alle die zahllosen Höhlengrabungen ergeben, die nicht nur in Frankreich (in der Dordogne, der Charente usw.), sondern auch z.B. in der Schweiz (CotencherHöhle; Dubois \& Stehlin 1933) und in Süddeutschland ausgeführt worden sind. Man denke z.B. an die Grabung von R. R. SснміDт (1912) im Sirgenstein mit ihrer Schichtfolge, die vom Moustier über Aurignac, Solutré zum Magdalen geht und die wieder stets nur kalte Faunen zeigt, wohl mit Abstufungen im einzelnen, aber niemals mit Einschaltung einer wirklich warmen Fauna.

Dasselbe gilt auch für die neuerdings von U. LeHMANN (1954) untersuchte Fauna vom Vogelherd im Lonetal. Uber warmzeitlichen Bildungen (mit Elephas antiquus) folgt ein bohnerzführender gelber Lehm mit Moustier und einer kalten Fauna (u. a. Elephas primigenius und Rhinoceros tichorbinus). Ein darüber liegender lehmiger Kalkschutt enthält Aurignac, wieder mit den beiden genannten Dickhäutern, ferner mit Rangifer, Gulo, Vulpes lagopus und Rupicapra. Man kann daraus mit Lehmann auf eine Verschärfung des kaltzeitlichen Charakters der Fauna schließen. Die obere Hälfte des nun fast reinen Kalkschuttes enthielt zwei Magdalen-Horizonte. Die Fauna ist nicht so umfangreich, enthält aber wieder Rhinoceros tichorbinus und Rangifer, ferner Ovibos moschatus und Equus przewalskii. Eine unbefangene Betrachtung der Faunenentwicklung läßt also im ganzen den Ubergang von einer warmen Fauna an der Höhlensohle über eine schon kalte im MoustierHorizont zu einer hocheiszeitlichen im Aurignac und Magdalen erkennen, d.h. eine Entwicklung, wie wir sie an zahllosen sonstigen Profilen beobachten. U. LeHMANN erörtert zwar die Frage, ob diese Folge durch eine Warmzeit (zwischen Moustier- und Aurignac-Horizont) unterbrochen gewesen sei. Doch liegt in den dortigen Beobachtungen keinerlei Anlaß für diese Annahme vor. 
Was an zahllosen Beispielen in Frankreich, Süddeutschland und anderen Gegenden nachgewiesen ist, das wird durch neue Untersuchungen in weiter östlich gelegenen Gebieten bestätigt. Dabei soll hier nicht auf Niederösterreich eingegangen werden, da dies in mehreren Arbeiten dieses Bandes behandelt wird, wohl aber kurz auf Böhmen und Mähren.

Das böhmisch-mährische Lößgebiet, von dem Syкora \& Urbanek (1955) eine Verbreitungskarte geben, ist in den letzten Jahren nach den verschiedensten Gesichtspunkten stratigraphisch-geologisch, bodenkundlich, nach dem Fossilinhalt, besonders den Mollusken, und nach den auftretenden Kulturen - untersucht worden (zahlreiche Arbeiten in " A n t h r o p o z o i k u m", Bd. I-IV; ferner R.Lais 1951, 1954). In fast allen Gebieten hat sich eine Dreigliederung des Würm-Lösses ergeben. Von den beiden auftretenden Interstadialen ist das ältere offenbar wärmer und von längerer Dauer gewesen. Es entspricht der Göttweiger Verlehmungszone in Niederösterreich. Das jüngere Interstadial ist, wie in Niederösterreich, nur schwach ausgebildet. Jede einzelne Lößstufe beginnt für gewöhnlich mit einem Solifluktionshorizont. Was die Mollusken anbelangt, so ist nach V. LožEK (1955) für das Riß-Würm-Interglazial die sog. "Banatica-Fauna“ (mit Helicigona banatica, Cepaea vindobonensis u.a.) bezeichnend. In der älteren Würm-Eiszeit, d.h. im unteren Jüngeren Löß, findet sich dann eine noch verhältnismäßig wärmeliebende Steppenfauna ("Striata-Fauna“ mit Helicella striata, Abida frumentum, Pupilla muscorum u.a.). Sie wird während der Sedimentation des mittleren Jüngeren Lößes von einer kühleren Fauna abgelöst, die im oberen Jüngeren Löß einen ausgesprochen kalten, boreo-alpinen Charakter annimmt ("Columella-Fauna" mit Columella edentula columella, Vertigo parcedentata, Fruticicola hispida u.a.). Wir haben also, wenn wir von den Interstadialen absehen, eine allmähliche Zunahme der Kälte, die ihr Maximum im Oberen Jüngeren Löß erreicht.

Die menschlichen Kulturen sind nach K. ŽEBERA (1955) in folgender Weise im böhmisch-mährischen Jüngeren Löß verteilt: Eine Moustier-Industrie (mit clactonartiger Abschlagtechnik) tritt im höheren Teil des unteren Jüngeren Lösses auf. Die Szeleta-Industrie mit Lorbeer- und Pappelblattspitzen, ovalen Schabern, vielfach aus Quarz und Quarzit, liegt an der Basis des Jüngeren Löß II, nach ProšEK \& LožEK $(1954,1955)$ im Waagtal (Slowakei) im Interstadial „I/IJ““. Nach K. ŽEEERA entspricht sie zeitlich etwa dem westlichen Châtelperron. Typisches Aurignac ("Aurignacien typique“, d.h. nach der älteren Bezeichnung: mittleres Aurignac) ist nach ŽEBERA aus dem böhmisch-mährischen Löß bisher nicht bekannt, wohl aber aus Oberflächenaufsammlungen in Mähren. Weit verbreitet ist dagegen im Löß die Gravette-Klingenindustrie mit ihren Messern und Spitzen mit abgestumpften Kanten. Stratigraphisch liegt diese Kultur an der Basis des Oberen Jüngeren Lösses bzw. in der Verlehmungszone „II/III“, in Lubná (Zentral-Böhmen) i n diesem obersten Jüngeren Löß (K. ŽEBERA 1952). Ein dem Gravette noch ziemlich nahe verwandtes Magdalen (mit kleinen Formen) tritt schließlich in Höhlen und Freilandstationen auf. In der Lößstratigraphie würde das etwa dem höchsten Teil des Jüngeren Lößes entsprechen.

So zeigt sich also überall eine Gliederung des Jüngeren Lösses durch Verlehmungszonen, Geröllhorizonte usw., ohne daß jeweils eine echte interglaziale Fauna und Flora aufträte. Wohl sind Unterschiede vorhanden, die auf vorübergehende Milderung des Klimas hinweisen, niemals aber schaltet sich eine echte Interglazialzeit ein. Auftreten und Unterbrechung der Lößbildung während der Letzten Eiszeit können wir durch das in Abb. 1 gegebene Schema verdeutlichen. Ich gehe dabei von der früher (1954) von mir gezeichneten Kurve der Letzten Eiszeit aus, die ich nur in einzelnen Punkten etwas abändere.

Die Kurve ist als „Vereisungskurve“ für Norddeutschland gezeichnet worden, die die jeweilige Entfernung des Eisrandes vom (theoretischen) Vereisungszentrum aus angibt. Als Grundlage dienen die verschiedenen Stadien der nordischen Weichselvereisung. Die Kurve ist gegenüber der früher gegebenen Darstellung noch stärker unsymmetrisch gezeichnet worden in der Annahme, daß der Vorstoß (unter ozeanischem Klima) länger gedauert 


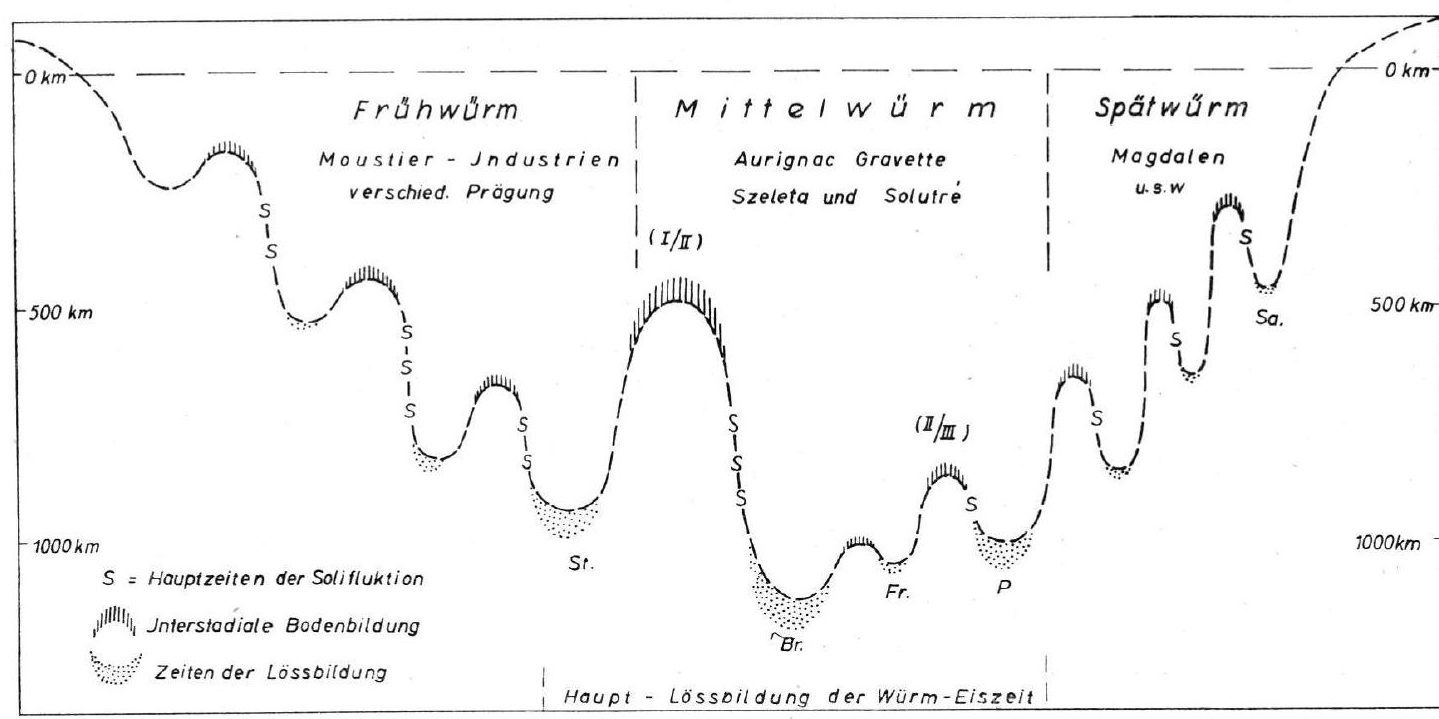

Abb. 1. Schematische Darstellung der Bewegung des Eisrandes (der Nordischen Vereisung), der Lößbildungs-, Solifluktions- und Bodenbildungszeiten während der Letzten Eiszeit in Mitteleuropa. St. $=$ Stettiner, Br. $=$ Brandenburger, Fr. $=$ Frankfurter, P. $=$ Pommersches, Sa. $=$ Salpausselkä-Stadium.

hat als der Rückzug (unter mehr kontinentalem Klima). Würde man eine reine Temperaturkurve zeichnen, so müßte diese wahrscheinlich gegenüber der Vereisungskurve verschoben gezeichnet werden derart, daß die stärkste Temperaturabsenkung gegen Ende von Mittelwürm, d.h. etwa beim Pommerschen Stadium, läge.

Lößbildung erfolgt im wesentlichen nur während der einzelnen Teilvorstöße, aus denen sich die Gesamtkurve zusammensetzt. In den Interstadialen erfolgt Verlehmung, beim Beginn der neuen Vorstöße Erdfließen, Kryoturbation usw. Die Zahl der einzelnen Lösse wird in den verschiedenen Gebieten nicht immer gleich sein. Wir kennen spätglaziale Jüngste Lösse, die nur ganz lokal verbreitet sind (vergl. u. a. E. SchöNhals 1944a, 1944b.) Ähnliches müssen wir für die frühe Phase der Würmvereisung annehmen. S o b r a u c h e n die lokalen Gliederungen nicht überall übereinzustimmen (vgl. z.B. die oben angeführte Gliederung von F. Bondes (1954) in Frankreich). Aber es hebt sich doch eine größere Gliederung in drei Abschnitte heraus, und zwar durch zwei über größere Gebiete zu verfolgende Verlehmungszonen.

Eine solche, besonders im Osten stärker entwickelte Verlehmungszone trennt einen Unteren Jüngeren Löß von einem Mittleren ab. Dieser Untere Jüngere Löß ist im Seinebecken nach F. BORDES wieder unterteilt; weiter im Osten erscheint er einheitlich. Er enthält die verschiedenen Moustier-Industrien. Sie sind besonders reich in Frankreich vertreten, sind aber neuerdings, wie schon gesagt wurde, auch z. B. in Předmost gefunder worden (K. ŽEBerA u. a. 1955). In das Interstadial („I/II“), das dicser ältesten Würm” phase ("Frühw ü rm ") folgt, gehören offenbar die Bodenbildungen von O ber F e ll a b r u n (F. Brandtner 1954), S ti $11 \mathrm{f} \mathrm{ried} \mathrm{A} \mathrm{(J.} \mathrm{Fink} \mathrm{1954)} \mathrm{und} \mathrm{ebenso} \mathrm{die}$ vielumstrittene Göt t w e i g e $\mathrm{B}$ od en bildung. Doch diese Fragen werden in den anderen Arbeiten dieses Bandes erörtert. In diesem Interstadial finden sich im Westen nach BORDES noch die ausgehenden Moustier-Industrien. Im Osten dagegen beginnen in ihm bereits die Szeleta- und Aurignac-Industrien. Sie charakterisieren den als $\mathrm{M}$ i t t e l w ü r $\mathrm{m}$ zu bezeichnenden Abschnitt, in dem wohl das Maximum der Eisausdehnung erreicht wird. Ein schwächeres Interstadial („II/III“ der früheren Bezeichnung) trennt den mittleren Jün- 
geren Löß vom oberen ab. Im Westen ist es als Solifluktionszone zwischen dem Jüngeren Löß IIIa und IIIb von F. BORDEs entwickelt; im Osten gehören die sog. P a u d o r f e r $\mathrm{H}$ u m u z o n e (G. Götzinger 1935, F. Brandtner 1954) und der Bodenkomplex Stillfried B (F. FINK 1954) in dieses Interstadial. Urgeschichtlich ist es im Osten charakterisiert durch das Gravette, das hier beginnt und sich dann weiter im oberen Jüngeren Löß findet. Das Magdalen tritt, wie früher schon gesagt wurde, erst oberhalb dieses oberen Lößes auf.

Das zwischen Früh- und Mittelwürm liegende Interstadial (in Abb. 1 als „I/II“ bezeichnet) wird von mehreren Autoren als recht warm und interglazialähnlich angesehen. F. Zeuner (1954), mit dem ich sonst in allen wesentlichen Punkten der Lößgliederung durchaus übereinstimme, glaubt, daß vielfach die Ablagerungen dieser Interstadialzeit mit denen des echten Letzten Interglazials verwechselt würden. Wer sich mit den Ablagerungen der Letzten Interglazialzeit, insbesondere auch mit den Pollendiagrammen eingehender beschäftigt hat, wird diese Ansicht nicht teilen können. Das Eem-Interglazial weist so einmalige charakteristische Züge auf, daß es schlechterdings nicht vorstellbar ist, derselbe Ablauf habe sich in allen Einzelheiten zweimal wiederholt. Im übrigen ist, soweit ich sehe, der Beweis bisher nicht erbracht worden, daß im Interstadial I/II eine wirklich interglazialähnliche Zeit vorläge. Über die Bodenbildung möchte ich mich hier nicht äußern. Das geschieht in diesem Bande von seiten mehrerer Bodenkundler. Auf das Fehlen einer wirklich wärmeliebenden Fauna ist oben mehrfach hingewiesen worden. Die sehr grünlichen Untersuchungen LožEK's (1955) über die Molluskenfauna zeigen nur, daß überhaupt der ganze erste Abschnitt (Frühwürm und Interstadial) noch nicht durch kalte Formen gekennzeichnet ist. Die wirklich eiszeitlichen kalten Formen folgen erst später.

Von F. Brandtner (1949) wird das Roggendorfer Niedermoor bei Melk (vgl. auch A. TAuber 1942) in die Interstadialzeit I/II gestellt. Diese Einordnung kann aber noch nicht als völlig gesichert gelten. Die von mir geäußerten Bedenken werden auch von H. Gross geteilt, der mir brieflich mitteilt, daß er die Moorbildung für spät- bzw. postglazial hält, weil sie ja nur teilweise von Löß (Schwemmlöß?) überdeckt ist, während ein anderer Teil unmittelbar an der Oberfläche liegt. Träfe aber die Einordnung des Moores in das Interstadial I/II zu, dann hätten wir in diesem Interstadial tatsächlich eine wärmere Phase, in der die Laubbäume begonnen hätten einzuwandern. Man wird die Prozentsätze, mit denen die Laubbäume und die Hasel im Roggendorfer Pollendiagramm auftreten, nicht gänzlich auf Ferneinwehung zurückführen können, wenn auch dies Gebiet den eiszeitlichen Refugien der Laubbäume bedeutend näher war als etwa Norddeutschland. Es hätte also - immer unter der Voraussetzung, daß das Roggendorfer Niedermoor in das Interstadial I/II gehört - der Beginn der Einwanderung der Laubbäume eingesetzt, der dann aber abgebrochen wurde durch wieder zunehmende Kälte.

BRANDTNER gibt auch sonst aus dem Fellabrunner Horizont das Vorkommen von Laubbaumpollen an. Sollten weitere Funde das bestätigen, so hätten wir zwar den Beginn einer Wärmezeit, niemals aber ein voll entwickeltes Interglazial, und wir würden auch dänn diesen Zeitraum immer besser als Interstadial, nicht jedoch als Interglazial zu bezeichnen haben.

Wir besitzen aber andere Untersuchungen, und zwar solche aus Ungarn, die ein klareres Bild des Interstadials W I/II zeigen. Die Untersuchungen der Holzkohlenreste aus der Istallóskö-Höhle im ungarischen Bükk-Gebirge (J. VÉRTES u. a, 1955) durch S. SÁRKÁNy \& J. Stieber ergab, daß unter knapp 900 Proben, die von den Verfassern in dies Interstadial gestellt werden, nur 9, d.h. 1\%, von Laubbäumen (Quercus, Acer und Fagus) stammten. Diese können von Refugien im Pannonischen Becken herkommen. Der Wald der Umgebung bestand jedenfalls im wesentlichen aus Pinus cembra, Larix und Picea. Pinus cembra überwiegt stark in der unteren und oberen Kulturschicht (mit Aurignac). In der dazwischen gelegenen Schicht tritt sie gegenüber Larix und Picea zurück. Wahr- 
scheinlich war dieser mittlere Abschnitt durch ein etwas wärmeres und feuchteres Klima charakterisiert. -

Eine äußerst wichtige Frage ist nun, wie ich schon 1948 betont habe, die: welchen Stadien der Vereisung entsprechen die verschiedenen Würm-Lösse? Diese Frage kann für das Nordeuropäische Vereisungsgebiet zunächst $\mathrm{nur}$ verm u t u g s w e is e gelöst werden. Den unteren Jüngeren Löß mit den vorhergehenden und eingeschalteten Kryoturbationszeiten sehe ich als Äquivalent der vorrückenden Weichselvereisung bis zum Stettiner Vorrückungsstadium ${ }^{3}$ ) an. Es folgt das Interstadial zwischen dem Stettiner und dem Brandenburger Stadium, in das möglicherweise ein Teil der Rixdorfer Fauna gehört, und das ich - bis zum Beweise des Gegenteiles - als Äquivalent der Göttweiger Bodenbildung ansehe. Wie weit damals der Eisrand zurückgewichen ist, wissen wir nicht. Brandenburger und Frankfurter Stadium dürften dem mittleren Jüngeren Löß entsprechen. Dieser Löß ist es, der hauptsächlich in der nördlichen Randzone des mitteldeutschen Lößverbreitungsgebietes vorhanden ist. Es sei z.B. an die Aurignacfunde im Leinetal (W. BARNER 1937) erinnert.

Es ist hier angenommen worden, daß der oberhalb der schwachen Paudorfer Verlehmungszone liegende Löß (bisher meist als „Jüngerer Löß III“ bezeichnet) dem Pommerschen Stadium entspricht. Das ist natürlich zunächst rein hypothetisch. Die Paudorfer Zone würde dann dem Interstadial vor dem Pommerschen Stadium entsprechen. Als über diesem Interstadial liegende Lösse wären möglicherweise die Flottlehme des Flämings und der Lüneburger Heide anzusehen.

Leider fehlt es in Norddeutschland an genügend zahlreichen paläolithischen Stationen, um mit ihrer Hilfe zu einem sicheren Ergebnis zu kommen. Aber das wenige, das vorhanden ist, spricht in dem oben dargelegten Sinne. Aus dem Letzten Interglazial, dem EemInterglazial, das zwischen Weichsel und Warthe liegt, haben wir den Fund von Le h$\mathrm{r}$ in g e $\mathrm{n}$, der Levalloisabschläge zusammen mit Elephas antiquus und einem Holzspeer aus Eibenholz erbrachte. Es ist dann die Moustier-Industrie von L e b e n s e d t bei Salzgitter zu nennen (vgl. die Ausführungen von A. Tode und Mitarbeitern in Bd. 3 dieses Jahrbuches). Sie fand sich in Solifluktionsschichten, die in die Frühwürmzeit zu stellen sind. Darüber liegen verschwemmte Jüngere Lösse, die wahrscheinlich dem Mittelwürm angehören.

Wir kommen schließlich zu der sehr wichtigen Frage, in welcher Weise die Würm-Eiszeit $\mathrm{zu}$ gliedern ist und welche Namen den verschiedenen Abschnitten zu geben sind. Dabei muß zunächst festgestellt werden, daß man für eine solche Einteilung und Bezeichnung keinesfalls allein die Lößgliederung verwenden kann. Denn die Lößbildung umfaßt ja nicht die ganze Eiszeit, sondern nur bestimmte Abschnitte, die zum größten Teil hocheiszeitlich sind. Es kommt ein langer frühglazialer und ein langer spätglazialer Abschnitt hinzu. Unter Berücksichtigung aller Umstände, wobei auch die eingeschlossenen urgeschichtlichen Industrien eine Rolle spielen, wird man zu folgender Einteilung kommen (die auch bereits ähnlich in einigen Arbeiten verwendet worden ist, z.B. von ED. EBERS 1955):

Ein erster Abschnitt wird als $\mathrm{Fr}$ ü h w ü $\mathrm{r} m$ bezeichnet. Er umfaßt den ganzen Vorstoßabschnitt, der besonders durch Fließerdebildung und Kryoturbation charakterisiert ist, dem aber auch der älteste Jüngere Löß angehört („Jüngerer Löß I“, der örtlich vielleicht unterzugliedern ist, z.B. nach F. Bordes im Seinebecken). Das Frühwürm enthält im wesentlichen die (kalten) $\mathrm{M}$ o u s t i e r - Industrien verschiedener Prägung. Der Abschnitt reicht bis zum Beginn des offenbar größeren Interstadials, das sich in vielen Lößgebieten durch eine stärkere Bodenbildung zu erkennen gibt. Dieses Interstadial wird selber bereits

3) Ich wähle diesen Namen, nicht die von J. Hesemann (1930) angewandte Bezeichnung: Rosenthaler Randlage, weil darunter damals ein Stadium $\mathrm{n} \mathrm{a} \mathrm{ch}$ dem Pommerschen verstanden wurde. 
zum Mit t e l w ü r m - Abschnitt gerechnet. In Norddeutschland wird, wie schon gesagt, die Schwankung zwischen Stettiner und Brandenburger Stadium hierher gestellt.

Wie aber soll man dies Interstadial, das in der Literatur meist unter der Bezeichnung „W I/II“ erscheint, benennen? Soll man von einem „Göttweiger“ oder „Fellabrunner Interstadial“ sprechen? Vielleicht wird man erst noch abwarten, wie weit sich das genannte Interstadial in den verschiedenen Gebieten nachweisen läßt, ehe man einen bestimmten Namen wählt. Den Ausdruck „F-Wärmezeit“, den F. BrandTneR (1954) vorschlägt, halte ich nicht für günstig, weil wir uns daran gewöhnt haben, den Begriff "Wärme-“ oder „Warmzeit“ für echte Interglazialzeiten zu verwenden, nicht aber für eine, wenn auch verhältnismäßig warme, Interstadialzeit.

Das M i t te l w ü rm umfaßt im übrigen den hochglazialen Abschnitt der Letzten Vereisung. Das Eis erreichte jetzt im allgemeinen seine größte Ausdehnung, und nun setzte der trockene und kalte Abschnitt ein, mit dem die Hauptlößbildung verknüpft war. In diesen Abschnitt dürfte die Hauptmenge des Lösses der Letzten Vereisung gehören. Diesen Löß hätte man in seiner Gesamtheit als „Jüngeren Löß II“ zu bezeichnen. Will man ihn (z.B. durch den Paudorfer Horizont) weiter untergliedern, so erscheint es am besten, von Jüngerem LößIIa und IIb zu sprechen, nicht aber von Jïngerem LößII und III.

Urgeschichtlich gehören in den Mittelwürm-Abschnitt in der Hauptsache das A u r i g $n$ a c und das Grave t $\mathrm{te}$, aber auch die Szelet a- und S olut r é-Industrien. Es scheint, daß das Jungpaläolithikum im Osten etwas früher auftritt als im Westen. In Mitteleuropa fällt dieser Zeitpunkt etwa mit dem Beginn des Hauptinterstadials zusammen, d.h. mit dem Beginn des Mittelwürm-Abschnittes. In Frankreich findet sich nach F. Bordes (1954) das allerletzte Moustier noch in der Solifluktionszone, die den nächsten Löß („Jüngerer Löß IIa“) einleitet.

Das $S \mathrm{p}$ ä $\mathrm{t} w$ ü $\mathrm{r}$, das man am besten mit der Wärmeschwankung nach dem Pommerschen Stadium beginnen läßt, umfaßt den bisher meist als "Spätglazialzeit“ bezeichneten Abschnitt. Urgeschichtlich ist es durch das $\mathrm{Mag} d$ a $\mathrm{l}$ e $\mathrm{n}$ und die mit ihm gleichaltrigen Kulturen charakterisiert.

Úber das alpine Gebiet sei hier nur soviel gesagt, daß als Frühwürm das Vorrücken des Eises bis zu einem der später überfahrenen Stadien angesehen wird. Das Mittelwürm würde mit der durch den „Innerwürmboden“ von F. KRAus (1955) bezeichneten größeren Wärmeschwankung beginnen und dann das Maximum umfassen bis zu den Rückzugsstadien, die etwa in der Nähe der überfahrenen Frühwürmphase liegen, das Spätwürm schließlich den ganzen weiteren Rückzug einschließlich der sog. Schlußvereisung.

Fassen wir das Gesagte zusammen, so zeigt sich, daß W. SoERGEL mit seiner Auffassung von einem gegliederten Jüngeren Löß durchaus Recht hatte. Sein Jüngerer Löß I enthält die Moustier-Industrien, die ohne jeden Zweifel in die Frühwürmzeit gehören, während sein Jüngerer Löß II durch das Aurignac charakterisiert ist. Wie die Ausführungen gezeigt haben, läßt sich diese Gliederung noch etwas verfeinern. SoERGEL irrte, wenn er seinen Jüngeren Löß I mit dem Warthe-Stadium Norddeutschlands bzw. mit der "Größten Vergletscherung “ der Schweiz parallelisierte. Aber das schmälert seine großen Verdienste um die Beziehungen zwischen „Lössen, Eiszeiten und paläolithischen Kulturen" in keiner Weise.

\section{$\mathrm{Sch}$ rif ten- $\mathrm{Nachwe}$ is}

Adam, K. D.: Der Waldelefant von Lehringen. - Quartär 5, S. 79-92. Bonn 1951.

Agafonoff, V., \& Malycheff, Vera: Le loess et les autres limons du plateau de Villejuif. Bull. Soc. géol. France (4) 29, 1929.

BARner, W.: Die jungpaläolithische Besiedlung des Landes zwischen Hildesheimer Wald und Ith. - Nachr. aus Niedersachs. Urgesch. 11, S. 1-58. Hildesheim 1937.

Bordes, F.: Les limons quaternaires du Bassin de la Seine. - Arch. Inst. Paléont. Humaine, Mém. 26, Paris 1954. 
BRANDTNER, F.: Die bisherigen Ergebnisse der stratigraphisch-pollenanalytischen Untersuchung eines jungeiszeitlichen Moores usw. - Archaeologica Austriaca 2, S. 5-32. Wien 1949. - Jungpleistozäner Löß und fossile Böden in Niederösterreich. - Eiszeitalter und Gegenwart 4/5, S. 49-82, 1954.

Breuil, H., \& KoslowsKi, L.: Etudes de stratigraphie paléolithique etc. - Anthropologie 41, 1931 , und 42, 1932.

BüDEL, J.: Die Klimaphasen der Würmeiszeit. - Naturwiss. 37, 1950.

Bigot, A.: Les terrasses pleistocènes du littoral du Cotentin. - Livre jubil. etc. Soc. géol. France 1930.

Dubois, A., \& Stehlin, H. G.: La Grotte de Cotencher, station moustérienne. - Mém. Soc. paléont. suisse 52/53, 1933.

Dubois, G.: Recherches sur les terrains quaternaires du Nord de la France. - Mém. Soc. géol. du Nord 8, I. Lille 1924.

Dubois, G., \& Firtion, F.: Esquisse de l'extension des limons loessiques en France. - Bull. Serv. Carte géol. d'Alsace et de Lorraine 3, S. 21-26. 1936.

Ebers, Edith: Hauptwürm, Spätwürm, Frühwürm und die Frage der älteren Würmschotter - Eiszeitalter und Gegenwart 6, S. 96-109. 1955.

FINK, A.: Die fossilen Böden im österreichischen Löß. - Quartär 6, S. 85-107. Bonn 1954.

Frfising, A.: Neue Ergebnisse der Lößforschung im nördlichen Württemberg. - Jahresh. geol. Abt. württ. statist. Landesamts 1, S. 54-59. Stuttgart 1951.

Götzinger, G.: Zur Gliederung des Lösses. - Verh. geol. Bundesanst. 1935, S. 126-132.

HesemanN, J.: Zur Geschiebeführung und Geologie des Odergletschers. - Jb. preuß. geol. Landesanst. 53, S. 70-84, 1932.

Kraus, E.: Zur Zweigliederung der südbayerischen Würmeiszeit durch eine Innerwürm-Verwitterungsperiode. - Eiszeitalter und Gegenwart 6, S. 75-95, 1955.

Ladriere, M.: Etude stratigraphique du terrain quaternaire du Nord de la France. - Ann. Soc. géol. Nord 18, S. 93-149, Lille 1890.

LaIs, R.: UUber den jüngeren Löß in Niederösterreich, Mähren und Böhmen. - Ber. naturf. Ges. Freiburg i. Br. 41, S. 119-168, 1951. - - Über den Löß von Unterwisternitz (Mähren). Palaeohistoria 2, S. 135-170. Groningen 1954.

Lehmann, U.: Die Fauna des "Vogelherds" bei Stetten ob Lontal (Württemberg). - N. Jb. Geol. Paläontol., Abh. 99, S. 33-146, Stuttgart 1954

LožEx, V.: Die Mollusken des tschechoslowakischen Quartärs (tschechisch mit russ. und deutscher Zusammenfass.) - Rozpravy Ustredniho ûstavu geologického, Svazek 17. 510 S. Prag 1955.

Prošek, Fr., \& LožEK, V.: Untersuchung des Lößprofils von Zamarovce bei Trencin (tschech., russ., deutsch). - Anthropozoikum 4, S. 181-211, Prag 1955.

SARKany, S., \& S STIEBER, J.: Anthrakotomische Bearbeitung der in neuester Zeit in der Höhle von Istállóskö freigelegten Holzkohlenreste. - Acta archaeol. Acad. Sci. hungar. 5, S. 211-233, Budapest 1955.

SснміDт, R. R.: Die diluviale Vorzeit Deutschlands. - Stuttgart 1912.

Schönhals, E.: Spätglazialer Löß in Lettland. - N. Jb. Min. etc., Mon.h. 1944 B, S. 241-250 [1944a]. - - Jungglazialer Löß auf Rügen. - Ber. Reichsamt f. Bodenforsch. Wien 1944 $[1944 b]$.

Soergel, W.: Lösse, Eiszeiten und paläolithische Kulturen. - Jena 1919.

Sykora, L., \& Urbanek, L.: Problems of building on loesses and loess soils (tschech., russ., engl.). - Anthropozoikum 4, S. 27-52. Prag 1955.

TAuber, A. F.: Ein jungeiszeitliches Niedermoor aus der Umgebung von Melk (Niederdonau.). Quartär 4, S. 109-124. Freiburg i. Br. 1942.

Tode, A., u. a.: Die Untersuchung der paläolithischen Freilandstation von Salzgitter-Lebenstedt. Eiszeitalter und Gegenwart 3, S. 144-220. 1953.

VÉrtes, L., u. a.: Die Höhle von Istállóskö. - Acta archaeol. Acad. Sci. hungar. 5, S. 111-291. Budapest 1955.

Weidenbach, F.: Zeitliche Einordnung der jungpleistozänen Ablagerungen Mitteleuropas. - Actes IV. Congr. Inqua Rome-Pise 1953. Rom 1955.

Woldstedt, P.: Saaleeiszeit, Warthestadium und Weichseleiszeit in Norddeutschland. - Eiszeitalter und Gegenwart 4/5, S. 34-48. 1954.

ŽEbera, K.: Conditions géologiques de la station gravettiene de Lubná près Rakovnik (tschech., russ., deutsch). - Anthropozoikum 2, S. 7-28. Prag 1952.

Žebera, K., u. a.: Bericht über die II. Etappe der Durchforschung des Quartärs in Předmosti bei Přerov in Mähren (tschech., russ., deutsch). - Anthropozoikum 4, S. 291-362. Prag 1955.

Zeuner, F. E.: Riss or Würm? - Eiszeitalter und Gegenwart 4/5, S. 98-105. 1954.

Manuskr. eingeg. 13. 2. 1956.

Anschrift d. Verf.: Prof. Dr. Paul Woldstedt, Bonn, Argelanderstraße 118. 